\title{
Preparedness and health risks associated with Moulay Abdellah Amghar moussem, Morocco, 2009-2010
}

M. Youbi, ${ }^{1}$ N. Dghoughi, ${ }^{1}$ M. Akrim, ${ }^{1}$ A. Essolbi, ${ }^{1}$ A. Barkia, ${ }^{2}$ A.I. Azami, ${ }^{3}$ A.T. Fleischauer, ${ }^{4}$ D. Schneider ${ }^{5}$ and A. Maaroufi

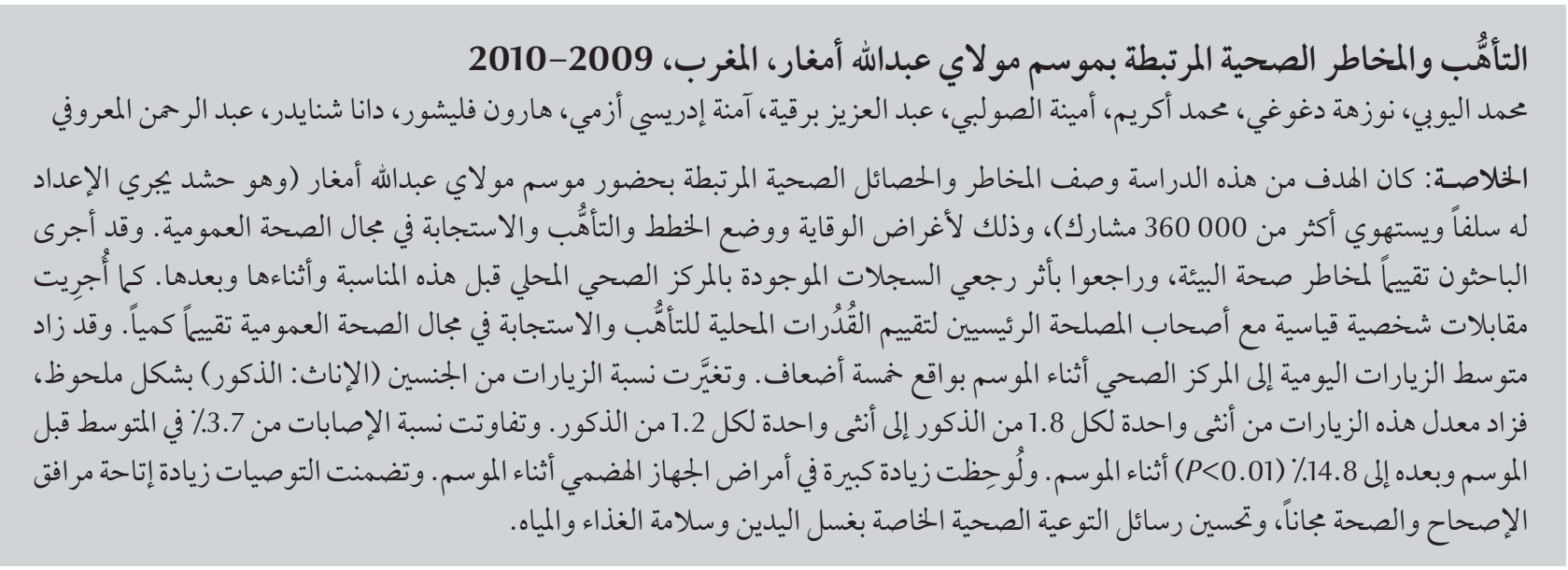

ABSTRACT The objective of this study was to describe the risks and human health outcomes associated with attendance at the Moulay Abdellah Amghar moussem (a pre-planned mass gathering attracting more than 360000 participants) for the purposes of public health prevention, planning, preparedness and response. We performed an environmental health risk assessment and retrospectively reviewed local health centre records before, during and after the event. In addition, standardized interviews with key stakeholders were performed to qualitatively evaluate local public health preparedness and response capacities. During the event, average daily health centre visits increased 5-fold. The sex ratio of health-care visits changed significantly from an average of 1.8:1 female:male visits per day to 1.2:1. The proportion of injuries varied from an average of $3.7 \%$ pre- and postevent to $14.8 \%(P<0.01)$ during the event. A significant increase in digestive diseases was also observed during the event. Recommendations include increasing accessibility to free sanitation and hygiene facilities and improving health communications concerning hand washing and food and water safety.

\section{Préparation et risques sanitaires associés au moussem de Moulay Abdellah Amghar (Maroc), 2009-2010}

RÉSUMÉ La présente étude avait pour objectif d'identifier les risques et les résultats sanitaires pour l'homme associés à la participation au moussem de Moulay Abdellah Amghar (un rassemblement de masse prévu à l'avance qui attire plus de 360000 personnes) à des fins de prévention, de planification, de préparation et d'action dans le domaine de la santé publique. Nous avons évalué les risques environnementaux pour la santé et avons procédé à un examen rétrospectif des registres des centres de santé locaux avant, pendant et après l'événement. Nous avons également réalisé des entretiens standardisés avec les principales parties prenantes afin d'évaluer, en termes de qualité, les capacités locales pour la préparation et la riposte en matière de santé publique. Pendant le rassemblement, le nombre moyen de consultations journalières dans les centres de santé a été multiplié par cinq. Le rapport de masculinité pour les consultations médicales a évolué de manière significative, passant d'une moyenne journalière de 1,8 femme pour 1 homme à 1,2 femme pour 1 homme. Le pourcentage des traumatismes est passé d'une moyenne de 3,7\%, avant et après l'événement, à $14,8 \%$ pendant le rassemblement $(P<0,01)$. On a aussi constaté une forte augmentation des maladies digestives pendant l'événement. II est recommandé, entre autres, d'augmenter l'accessibilité des installations gratuites d'assainissement et d'hygiène et d'améliorer la communication en ce qui concerne l'hygiène des mains et la sécurité sanitaire de l'eau et des aliments. 


\section{Introduction}

Moussems are an integral part of Moroccan culture. They are pre-planned, public, mass gathering events held for religious, social and cultural, and/or commercial purposes in urban and rural sites throughout the country. The term "moussem" comes from the Moroccan Arabic dialect and is related to the word "mawssim", which, in classical Arabic, means "season". Moussems are typically of short duration, held seasonally, and usually commemorate a saint. There are more than 300 annually, and the timing of many of these events corresponds to agricultural seasons (e.g. ploughing or harvest), well-known social events (e.g. the annual return of Moroccans who work abroad, holidays), and celebration of culture and art (e.g. festivals of roses, festival of engagements, spiritual music events).

The Moulay Abdellah Amghar moussem, one of the largest events, annually attracts more than 360000 people from across Morocco and internationally for a period of 8 days. It is held in the summer in El Jaddida province, Doukkala-Abda region, in the western part of the country on the Atlantic coast. This moussem is held in honour of a religious saint, Moulay Abdellah, and includes music, sport, cultural and religious activities, and fantasia, traditional horse shows in which riders perform military re-enactments with firearms that use powder (gunpowder) explosive.

Although there have been anecdotal reports of acute illnesses, outbreaks, and injuries associated with attendance at a moussem, the reports are typically not validated or surveyed by health officials. Environmental health risks and human health outcomes associated with mass gatherings have been well documented, and include hazards resulting from crowding, traffic, violence and terrorism, adverse weather exposure, unlicensed food and water vendors, and poor access to sanitation and hygiene facilities [1,2]. Additionally, healthcare facilities may not have appropriate surge capacity to manage these events. Information on human health outcomes would help to inform event-based preparedness and response efforts; however, no systematic evaluation of health risks and outcomes associated with moussems has been performed in Morocco [3].

The objectives of this study were to describe the specific health risks and morbidity associated with the Moulay Abdellah Amghar moussem and to evaluate the healthcare infrastructure and public health preparedness capacity for this event.

\section{Methods}

We conducted a retrospective study to describe the human health risks and outcomes associated with attendance at the Moulay Abdellah Amghar moussem. To accomplish the study objectives, we compared healthcare encounters before, during, and after the mass gathering from health centre medical records. Additionally, we conducted standardized interviews with key stakeholders to characterize the organizational and health preparedness aspects of this moussem.

We reviewed medical records of all patients seen at the health centre in Moulay Abdellah Amghar during the pre-event period ( -14 days to -7 days), the 8 days of the event and the postevent period $(+7$ days to +14 days) for the past 2 annual moussems (2009 and 2010). periods were selected because they are outside the period when attendees congregate at the event site (visitors begin to arrive several days before the event and stay several days after). Health centre visits were categorized as digestive disorders (acute gastrointestinal symptoms and/or gastroenteritis), acute respiratory disorders, other infectious diseases (e.g. pharyngitis, sexually transmitted diseases, rash, conjunctivitis, fever of unknown origin), heat-related illness (e.g. heat exhaustion, sunburns, sun stoke, and dehydration), scorpion bites, accidents attributable to the fantasia (e.g. falls from horses, injuries from the explosives), violence, road traffic injuries, burns, jellyfish stings, and drowning. All other visits such as chronic conditions were classified as "other."

A semi-structured questionnaire was administered to key stakeholders including health workers (provincial directorate, hygiene team health centre), an official from the Economic and Control unit of the Ministry of the Interior at the provincial level, representatives of the moussem organizers and the captain of the provincial civil protection unit. Questions included characteristics of the moussem, social and behavioural risks (e.g. animal sacrifice, ritual dancing in a state of trance, and other rituals specific to the Moulay Abdallah Amghar moussem), oganizational attributes, and the health system structure in place.

\section{Data analysis}

Medical records and questionnaire data were collected and entered into a Microsoft Excel spreadsheet. Statistical testing using the chi-squared test to compare proportions was performed using Epi Info, version 6.

\section{Ethical considerations}

All aspects of this study, including access to medical records, were approved by the Ministry of Health of Morocco. Interviews were conducted after having obtained informed consent. Information obtained from medical records and other health service providers was kept strictly confidential and no names or other identifying information on patients was collected. The key stakeholders and their supervisors were contacted in advance to explain the objectives of the study and official permissions were obtained prior to the interviews. 


\section{Results}

\section{Health outcomes associated with the Moulay Abdellah Amghar moussem}

The average number of health centre visits during the moussem period in 2009 and 2010 was 1577 per year. While the average daily number of visits was 36 outside the moussem period, it increased to 197 during the event.

The average daily ratio of female to male health centre visits during the moussem was 1.2. This ratio in the preand post-event time periods was 1.8 , indicating an increase in health centre visits by males during the moussem.

Among the 3153 health centre visits during the event in 2009 and 2010, $704(22 \%)$ were potentially due to an infection other than a respiratory or gastrointestinal illness, 467 were classified as accidents and injuries, 448 were for digestive disorders, 188 were likely an acute respiratory illness (Figure 1). Other chronic and acute conditions accounted for the unclassified ("other") health centre visits (42\%).

Comparing the average for the pre- and post-event time periods with the event time period, a significant increase was observed during the event for digestive disorders (10.9\% pre- and post-event vs $14.2 \%$ during the event, $P<0.05)$ and accidents and injuries (3.7\% vs $14.8 \%, P<0.01$ ). In contrast, a significant decrease was recorded for the number of acute respiratory disorders $(9.4 \%$ vs $6.0 \%, P<0.001)$ (Figure 1$)$.

During the moussem, men were nearly twice as likely to be injured or involved in an accident compared with women [331 (70.9\%) visits vs 136 (29.1\%) visits, $P<0.01$ ], while women were more likely to be seen for digestive disorders [286 (63.8\%) visits vs 162 $(36.2 \%)$ visits, $P<0.01]$ and infections other than acute gastrointestinal or respiratory infections [ $402(57.1 \%)$ vs 302 $(42.9 \%), P<0.01]$.

\section{Risk assessment and event planning analysis}

The Moulay Abdellah Amghar moussem typically attracts approximately 360000 national and international attendees each year for 8 days in July or August. Approximately 40000 (11\%) of these stay in the area for the duration of the moussem either, in tents or in rented

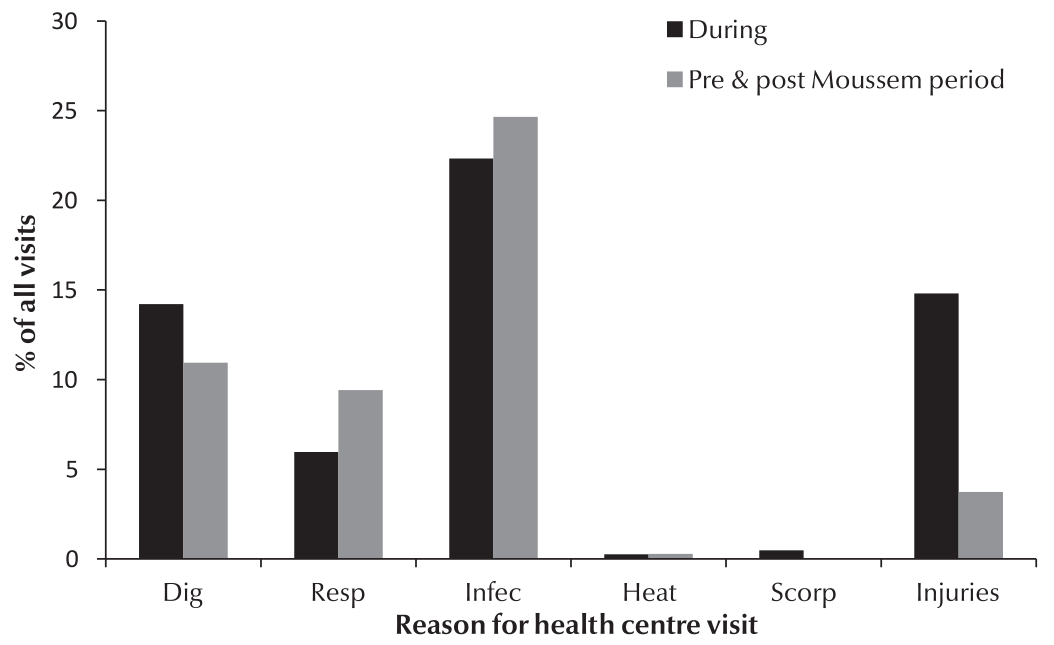

Figure 1 Reasons for health centre visits pre-event, post-event and during the Moulay Abdellah Amghar moussem, 2009 and 2010 (Dig = digestive disorders; Resp = acute respiratory disorders; Infect = other infectious diseases; Heat = heatrelated illness; Scorp = scorpion bites; visits for reasons classified as "other", e.g. chronic conditions, are not shown on this chart) houses. The moussem is hosted in a small rural village of 9000 residents, located on the coast near a small fishing port. The site is only accessible by a single $10 \mathrm{~km}$ secondary road (1 lane in either direction) from a main highway.

Potable well water is provided by water fountains equipped with many faucets; the well water is treated and monitored by a hygiene team from the Ministry of Health. However, private unregulated vendors selling well water can be found throughout the site. Bathroom and shower facilities are available for a fee, but few people use them because of the cost. Many attendees relieve themselves outdoors without the use of hand washing stations. Food is prepared by the attendees themselves in their tents and rented rooms. However, many small restaurants are available on site, often without the minimum requirements for food storage and conservation, including refrigerators.

Solid waste is handled by the city's waste management service and garbage collection is carried out twice a day. A private company is contracted to provide insect and rodent control. The hygiene team from the Ministry of Health, in collaboration with the Ministry of the Interior and the Ministry of Agriculture, inspect and manage the animal slaughter facilities and ensure food safety.

The local health centre is transformed into an emergency care facility during the moussem and operates 24 hours a day during the event. This health centre has 4 beds for short-term observation. Three clinical teams, each consisting of 2 general practitioners and 4 nurses, work 8-hour shifts. During the moussem, the health centre is equipped with surge capacity of common pharmaceuticals and medical equipment. In addition, 2 ambulances and staff are co-located with the health centre.

Planning for the moussem begins each year in January and is managed and coordinated by a provincial committee led by thegovernor of El Jadida province. 
The provincial committee designates 7 specific commissions (Equipment and Site Management, Fantasia and Folklore, Safety and Security, Surveillance and Consumer Protection, Heath and Hygiene, Communication and Coordination, and Religious Activities). These commissions are charged with all organizational and planning aspects. The Health and Hygiene Commission is supervised by the Ministry of Health. Coordination is done through meetings and periodic reports sent to the governor. During the moussem, the event site is divided into 6 areas, each of which is overseen by the head of the local authority (caid). A coordinating centre is established and daily meetings are held to discuss and assess the activities and problems.

\section{Discussion}

Moussems are culturally important and widely popular mass gatherings held throughout Morocco. This was the first study to conduct a risk assessment and identify human health outcomes associated with a moussem, specifically Moulay Abdellah Amghar, the most popular one in Morocco.

The finding that there was an increase in the average number of daily visits to the health centre confirms the large increase in workload during the event and amply justifies the implementation of a continuous, 24-hour, service.

The sex ratio of persons seeking healthcare has changed significantly in favour of males during the moussem, suggesting a high male predominance among the attendees. This finding could explain the increase in the proportion of health centre visits for accidents and injuries during the event. In fact, a large proportion of these accidents were related to falls from horses and from exploding firearms during the fantasia performances, which are exclusively male activities. Several other accidents and injuries, such as drowning and intentional injuries, are much more attributed to males and could be explained by the use of drugs and alcohol reported by all interviewed stakeholders in our study.

We also found that during the moussem, digestive disorders increased overall, particularly among females. This was likely a result of poor sanitation or water and food contamination, despite the sanitation and hygiene measures implemented by the organizers. In fact, the supply sources of private water vendors, as well as storage and distribution conditions, may be very suspect. Other aspects of hygiene may also be a source of suspicion, especially preparation of food in the many small restaurants on the site and their storage conditions. The most important source of suspicion could be, however, the limited access to sanitation facilities because they are only available for a fee; this would have lead to deficiencies in personal hygiene, including a lack of hand washing.

In this study, we expected greater proportion of digestive disorders during the moussem compared with accidents and injuries, but surprisingly, this was not the case. This finding is concordant with a descriptive study of all moussems in Morocco that was conducted in parallel to this one [Akrim M et al., unpublished report]. Otherwise, it is possible that the number of cases reported during the event was a significant underestimate since the incubation period of many acute illnesses is longer than the duration of time spent at the moussem.

\section{Limitations}

The quality of our results may have been affected by certain limitations, especially in relation to the information abstracted from medical records. Firstly, the reasons for health centre visits (e.g. complaints and diagnoses) were recorded by the clinical teams using different medical notations and abbreviations that do not refer to any specific criteria or uniform standards. Secondly, no international standard was used to validate illnesses and injuries categories for classing patients during data compilation for our study; therefore, selection bias could have been introduced. Thirdly, we were unable to epidemiologically link the reasons for the visits to the health centres absolutely to the event, and specifically we were unable to confirm etiologies for most acute illnesses.

Additionally, and owing to the lack of reliable data on the population distribution of the attendees, we were unable to calculate specific incidence rates of different health problem categories, especially by age and sex, in order to compare them with outside the event.

Despite these limitations, the results of our study allow us to make some recommendations for the attention of decision-makers, moussem organizers and officials of the health system for better preparedness and management of the event with a view to minimizing health risks.

\section{Recommendations}

To minimize the risk of acute gastrointestinal illness during moussems, we recommend that the health and hygiene committee enhance their public health interventions targeting hygiene and sanitation, especially through increasing accessibility to sanitation and hygiene facilities and making such facilities free to the public.

Unregulated water sellers should be prohibited. If this proves to be impossible, their sources must be identified and treated and containers should be checked and treated if necessary.

Small restaurants and food vendors should also be controlled and particular attention should be paid to the conditions of preparation as well as storage. Connection of their facilities to electricity should be provided by the organizers and the availability of refrigerators should be mandatory. 
These measures should be complemented by appropriate health education on hygiene of water and food, particularly on hand washing. A variety of communication channels should be used, such as interpersonal communication, audio messages and pamphlets. The installation of a dedicated moussem radio station could be useful for the dissemination of health education messages.

To reduce the number and severity of accidents and injuries, safety and security measures need to be strengthened. They should focus on the regulation of access to the site and the management of the public, traffic control and maintaining permanent access for emergency vehicles, prevention of risks related to the use of gas and electric equipment and facilities [4]. Special attention should be paid to improving safety related to the organization of fantasia shows, in particular storage conditions of explosive powder and loading guns.

All these measures should be planned during the preparation phase while providing simulations to test and make the necessary improvements, according to World Health Organization recommendations [5].

Additionally, an annual risk assessment prior to and during the moussem should be conducted in order to identify potential health hazards and to implement specific public health interventions.

In this context, and having regard to the intensity of the workload at the health centre during the event, it is recommended that health authorities strengthen the systems in place, including both human and material resources. Particular attention should be given to drugs and equipment needed to care for trauma and injury.

Because of the difficulties we experienced understanding the information contained in the health centre registers, we recommend implementing a surveillance system using appropriate surveillance forms and specific case definitions to rapidly identify and respond to adverse health outcomes during this moussem [6].

\section{Acknowledgements}

This study would not have been possible without the support and help of Dr N. Ben Chama, Chief of Medicine, Provincial Service of Infrastructure and Ambulatory Activities, and Interim Chief of Medicine, Ministry of Health Delegation of El Jadida province. We also wish to thank Mr Allal Mechbouh and Mme Soumya Belhamdounia from SIAAP, Mr Tahar Halam, Chief of service "Action Economique et de Contrôle”, El Jadida province, Le Capitaine Kamal, Commander of "Campanie provinciale de la Protection Civile", Dr Mohammed Kanar, Chief of Medicine, My Abdellah Health Centre, Mr Mohammed Erramch, Major, My Abdellah Health Centre and Mr Abderrahmane Moustaïd, SIAAP driver, El Jadida.

\section{References}

1. Ahmed QA, Arabi YM, Memish ZA. Health Risks at the Hajj. Lancet, 2006, 367:1008-1015.

2. Lucas N. Surveillance épidémiologique de l'Armada de Rouen [Epidemiological surveillance of Rouen Armada]. Saint-

3. Maurice, France, Institut de Veille Sanitaire, 2004 (http:// www.invs.sante.fr/publications/2004/armada_rouen/armada.pdf, accessed 20 September 2013).

4. Kadiri $\mathrm{N}$ et al. Morocco. In: Francoer RT, Noonan RJ, eds. The Continuum Complete International Encyclopedia of Sexuality. New York, Continuum International Publishing Group, 2004.

5. Memento manifestations publiques [Memento public events]. Puy-de-Dôme, France, Sapeurs Pompiers,2010 (GSMOO/ GSPR Version $\mathrm{N}^{\circ} 3$ ).
6. Rassemblements mondiaux de masse : répercussions et opportunitéspour la sécurité sanitaire mondiale, Rapport du Secrétariat [Global mass gatherings: implications and opportunities for global health security, Report by the Secretariat]. Geneva, World Health Organization, 2012 (A65/18) (http://apps.who. int/gb/ebwha/pdf_files/WHA65/A65_18-fr.pdf, accessed 20 September 2013).

7. Surveillance for early detection of disease outbreaks at an outdoor mass gathering - Virginia, 2005. Morbidity and Mortality Weekly Report, 2006, 55(3):71-74. 